\title{
Ocular rosacea
}

\section{Rosácea ocular}

Christian R. Fau ${ }^{1,2 *}$, Solange Nabzo ${ }^{1}$ and Veronica Nasabun ${ }^{3}$

${ }^{1}$ Ophthalmological Foundation 2020, Iberoamerican Cochrane Network; ${ }^{2}$ School of Medical Technology, Universidad Santo Tomás; ${ }^{3}$ Nursing School, Universidad Andrés Bello. Santiago, Chile

The patient is a 71-year-old female who lives in a rural sector, where wood heating and clay ovens are widely used. She has rosacea with facial telangiectatic erythema and ocular involvement of both eyes: blepharitis and meibomian gland dysfunction.

Three years ago, she presented a severe scleritis in the right eye that generated scleral thinning in several areas, in addition to a temporal corneal ulcer with corneal thinning and neovascularization (Fig. 1). Currently, she has an ocular reactivation, with a new episode of corneal ulcer in the same area affected 3 years ago (Fig. 2).

Rosacea is a disease of unknown etiology, with a prevalence of approximately $10 \%$ in the general population, and despite being mainly a dermatological disorder, in more than $50 \%$ of cases ocular manifestations occur; it is more frequent in women over 30 years of age and with white skin.

Although it is well known that this disease causes blepharitis with meibomian gland dysfunction and deficiency of the lipid layer of the tear film, it is forgotten that it can induce some serious complications: neovascularization in $11 \%$ of cases, episcleritis in $4.5 \%$,

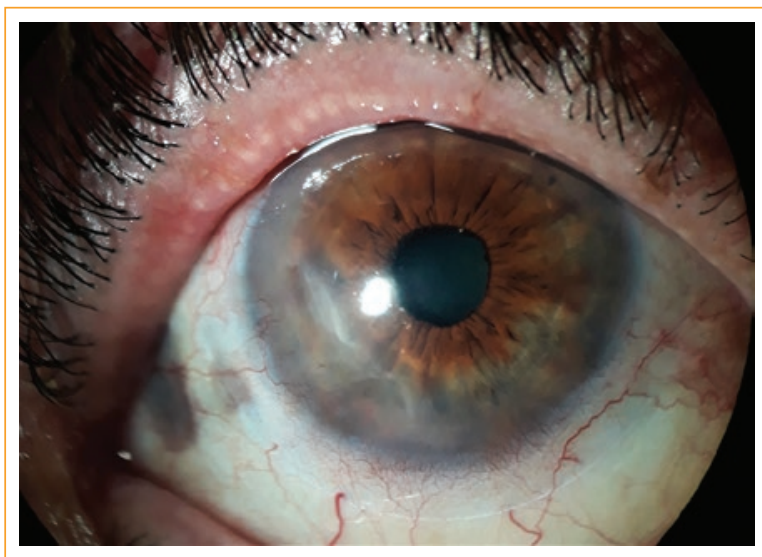

Figure 1. Several areas of scleral thinning and a large area of corneal thinning with neovascularization in the temporal sector of the right eye. The patient is wearing a Night $\&$ Day Aqua Air Optix contact lens.

corneal ulcer in $3.5 \%$, scleritis in $1.2 \%$ and corneal thinning in $5.7 \%$.

This clinical case reminds us on how important it is not to consider rosacea a trivial or low-risk disease, 


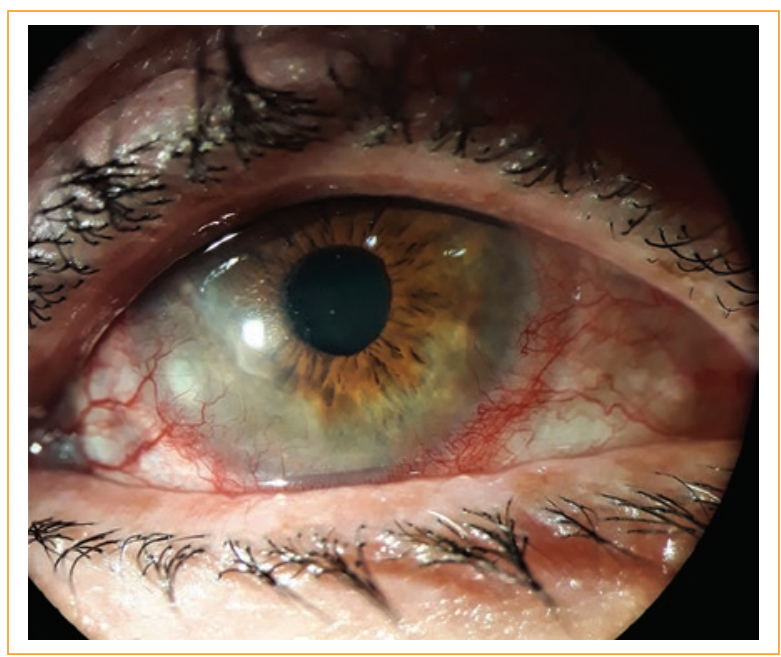

Figure 2. Reactivation of the corneal ulcer, with the same damaged and thinned area of the cornea as 3 years ago. The patient is wearing a Night \& Day Aqua Air Optix contact lens.

underestimate its risk factors, not inform triggers or aggravating factors, and finally under-treat the disease, thereby exposing patients to develop serious complications.

\section{Conflicts of interest}

The authors declare no conflicts of interest.

\section{Ethical disclosures}

Protection of human and animal subjects. The authors declare that the procedures followed were in accordance with the regulations of the relevant clinical research ethics committee and with those of the Code of Ethics of the World Medical Association (Declaration of Helsinki).

Confidentiality of data. The authors declare that they have followed the protocols of their work center on the publication of patient data.

Right to privacy and informed consent. The authors have obtained the written informed consent of the patients or subjects mentioned in the article. The corresponding author is in possession of this document.

\section{References}

1. Ghanem VC, Mehra N, Wong S, Mannis MJ. The prevalence of ocular signs in acne rosacea: comparing patients from ophthalmology and dermatology clinics. Cornea. 2003;22(3):230-3. 